\title{
Acoustically penetrable sonic crystals based on fluid-like scatterers
}

\section{A. Cebrecos}

Instituto de Investigación para la Gestión Integrada de zonas Costeras, Universitat Politècnica de València, Paranimf 146730 Grao de Gandia (Spain)

\section{Romero-García}

LUNAM Université, CNRS, LAUM UMR 6613, Av. O. Messiaen, 72085 Le Mans

(France)

E-mail: virogar1@gmail.com

\section{R. Picó, V. J. Sánchez-Morcillo}

Instituto de Investigación para la Gestión Integrada de zonas Costeras, Universitat Politècnica de València, Paranimf 146730 Grao de Gandia (Spain)

\section{Botey, R. Herrero, Y. C. Cheng}

Departament de Física i Enginyeria Nuclear, Universitat Politècnica de Catalunya, Colom 11, 08222 Terrassa (Spain)

\section{K. Staliunas}

Departament de Física i Enginyeria Nuclear, Universitat Politècnica de Catalunya, Colom 11, 08222 Terrassa (Spain). Institució Catalana de Recerca i Estudis Avanąts (ICREA)

\begin{abstract}
We propose a periodic structure that behaves as a fluid-fluid composite for sound waves, where the building blocks are clusters of rigid scatterers. Such building-blocks are penetrable for acoustic waves, and their properties can be tuned by selecting the filling fraction. The equivalence with a fluid-fluid system of such a doubly periodic composite is tested analytical and experimentally. Because of the fluid-like character of the scatterers, sound structure interaction is negligible, and the propagation can be described by scalar models, analogous to those used in electromagnetics. As an example, the case of focusing of evanescent waves and the guided propagation of acoustic waves along an array of penetrable elements is discussed in detail. The proposed structure may be a real alternative to design a low contrast and acoustically penetrable medium where new properties as those shown in this work could be experimentally realized.
\end{abstract}

Submitted to: J. Phys. D: Appl. Phys. 


\section{Introduction}

The propagation of acoustic waves through an inhomogeneous medium made of $N$ obstacles or scatterers embedded in a fluid is a broad topic with applications in different fields $[1,2]$. Such obstacles can be considered either as penetrable [3] or not penetrable [4]. Also, their spatial distribution can be ordered [5], as in some artificial materials as e.g. photonic or sonic/phononic crystals, or disordered [6], as some complex media found in nature as bubbly fluids or fish schools. It is general a complex problem involving many variables, but on the other hand it offers many possibilities to engineer wave propagation or to extract information about the medium.

During the last years, attention has been mostly paid to periodic systems, presenting unique dispersion properties. Those are mainly photonic crystals [7] for electromagnetic waves, sonic and phononic crystals [8, 9] for acoustic and elastic waves respectively, as well as periodic media for more exotic waves (like plasmons, Bose Einstein condensates (BECs) etc.) have been also considered.

The paralelism with electromagnetic waves is often used to predict new phenomena in acoustics, based on the analogy between the theoretical description of both systems, since both waves obey, in some limits, similar scalar wave equations. It has been the case, for example, with bandgaps, negative refraction, self-collimation, etc.

Photonic cystals can be built to present low or high index contrast, using dielectric or metallic elements respectively. The former represents the case of a penetrable system, where waves can propagate inside the scatterer and resonate within it, while the latter is a case of not penetrable system, where the wave propagates only in the host medium. Most of the studies in optics have considered dielectric crystals, where applications based on the phenomena mentioned above have been proposed. In acoustics, contrarily, the interest has been focused mostly on the case of rigid scatterers [10], with a high impedance contrast with the surrounding medium, and less attention has been paid to the case of penetrable scatterers. However, the relevance of penetrable scatterers in acoustics is recovering a renewed interest, where applications as enhanced transmission through gratings [11] or the control of waves using arrays of penetrable cylinders in waveguides [12] have been recently proposed. Special interest appears for the case of low contrast systems [13].

Solid scatterers can be assumed to behave as rigid elements for acoustic waves when its reflectivity, which depends on the contrast of impedances of the scatterer and the host material, is high. On the contrary, if the impedance contrast is low (for example, the case of $\mathrm{Al}$ in water), a substantial part of the wave can propagate through the scatterer, which are considered as penetrable elements. In acoustics, this brings some additional complexities. Penetrability may break the analogy with the case of electromagnetic waves, since as the wave penetrates in the solid the sound-structure interaction becomes

relevant, and transverse waves, corresponding to vibrations modes of the scatterers, can be easily excited, something like that has no analogue in electromagnetic wave systems. As a consequence, the assumption of longitudinal wave propagation becomes no more 
valid, and the dispersive characteristics of the system are altered [14].

Strictly speaking, dealing with only scalar sound waves under conditions of penetrability requires the use of fluid-fluid systems, i.e., fluid scatterers embedded in a fluid host. However, fluid-fluid composites present some drawbacks that makes them difficult to design, and are hardly realistic for experimental studies. There are still some proposals like composites made of mercury and water disposed in a boron nitride lattice, as presented in Ref. [9]. Other proposal consists of inserting a fluid inclusion into a latex bladder, but it is difficult to keep the structural integrity, and also some resonances can appear, arising from the elastic latex shell, that can perturb the properties of the system. Another possibility to implement such systems is to use porous scatterers with low absorption embedded in air, since porous materials can be treated as fluid-like materials over a wide range of frequencies [15]. However, the absorption due to the viscothermal losses, even being small, represents an inconvenient because they tend to drastically reduce the phenomena due to periodicity [16].

Here we show a physically realizable proposal to build a fluid-like, acoustically penetrable periodic system, overcoming some of the limitations of the above proposal. In the long wavelength regime, homogenization theories applied to periodic arrays of rigid inclusions embedded in a fluid medium allow to obtain the effective properties of the composite $[17,18]$. In this regime, a cluster of rigid cylinders embedded in a fluid behaves like an equivalent fluid with effective mass density and sound velocity determined by the filling fraction of the cluster with small losses [19]. In this work, we propose a novel acoustic structure formed by a periodic distribution of such fluid-like scatterers in a fluid host medium. The resulting composite becomes a powerful alternative to develop fluid-fluid composites with high tunable properties of the medium within the penetrable scatterer. Structures based on a similar bottom-up approach, with an effective value of the permeability in the visible spectral domain, have been recently proposed for optical waves $[24,25]$.

In the first part of this work, in Sec. 2.1, we present the structure made of penetrable fluid-like structures and the analytical and numerical techniques used to study its behaviour. In Sec. 2.2 we analyze both the scattering and the eigenvalue problems of a composite, and compare with the experimental results with a structure designed according the discussed theoretical assumptions. We find a good agreement that validates the main hypothesis. As an example of applicability of the proposed structure, in the remaining sections we present the acoustic analogues of two photonic phenomena: the focusing of unlocked evanescent waves [20] in Sec. 3 and the coupled resonator waveguide [23] in Sec. 4. Finally the summary and conclusions of the work are discussed in Sec. 5. 


\section{Acoustically penetrable sonic crystals}

\subsection{The penetrable structure}

The proposed fluid-like structure consists of a periodic arrangement of clusters (large scale (mesoscopic) scatterers) made itself of a periodic distribution of $N$ identical rigid scatterers, all embedded in a fluid. In the long wavelength regime $\left(\lambda>>a_{s}\right.$, being $\lambda$ the wavelength and $a_{s}$ the distance between the rigid scatterers within the cluster) the cluster can be considered as a fluid-like cylinder for acoustic waves with the following effective properties [19]

$$
\begin{aligned}
c_{e f f} & =\frac{c_{0}}{\sqrt{1+f_{s}}}, \\
\rho_{e f f} & =\rho_{0} \frac{1+f_{s}}{1-f_{s}},
\end{aligned}
$$

where $\rho_{0}$ and $c_{0}$ are the density and sound velocity in the host material respectively and $f_{s}=S_{s} / S_{c}$ is the filling fraction of the cluster. $S_{s}$ is the total area filled by the scatterers and $S_{c}$ the area of the cluster. Therefore, by adjusting the filling fraction of the cluster, one can change its mesoscopic physical properties such as the density and the wave velocity.

A possible scheme of a penetrable sonic crystal is shown in Fig. 1, where the scatteres are cylindrical clusters, each one formed by a small-scale periodic distribution of rigid square-rod scatterers. The system is embedded in a fluid. In this example, we have chosen the cylindrical clusters made of square rod scatterers, but it is worth noting that both the clusters and the scatterers can present different shapes. In this kind of structure the periodicities at both scales are characterized by two different lattice constants and two filling fractions. In the particular case shown in Fig. 1 we define the lattice constant, $a$, and filling fraction, $f=\pi R^{2} / a^{2}$, related to the arrangement of the cluster of radius $R$ defining the sonic crystal, and the lattice constant, $a_{s}$, and the filling fraction $f_{s}=l_{s}^{2} / a_{s}^{2}$ corresponding to the periodic structure of rigid square-rod scatterers with side length $l_{s}$. If the condition $a>>a_{s}$ is satisfied, the phenomena related to both scales of periodicity are uncoupled. Under these conditions, for the range of frequencies in which $\lambda \simeq a$, the mesoscopic structure behaves like a fluid-fluid band gap material made of fluid-like clusters which properties can be easily tuned by changing $f_{s}$ using Eq. (1) and (2). The main feature of the system is that it has double-periodicity, one in the long wavelength regime which fixes the physical properties of the equivalent fluid of the clusters, and other one in the strongly dispersive regime that fixes the Bragg frequency and, more generally, the dispersion relation.

Through this work three different techniques have been used to evaluate the dispersion relations (eigenvalue problem) for different structures. We will solve both the $\omega(\mathbf{k})[26]$ and the $k(\omega)[27,28]$ problems by means of different techniques based on plane wave expansion (PWE) [26, 27, 28] and finite element methods (FEM) [29]. In the case of periodic eigenvalue problem, the properties of the Bloch states constrains the solution to a unit cell with Bloch vectors in the first Brillouin zone. On the other 


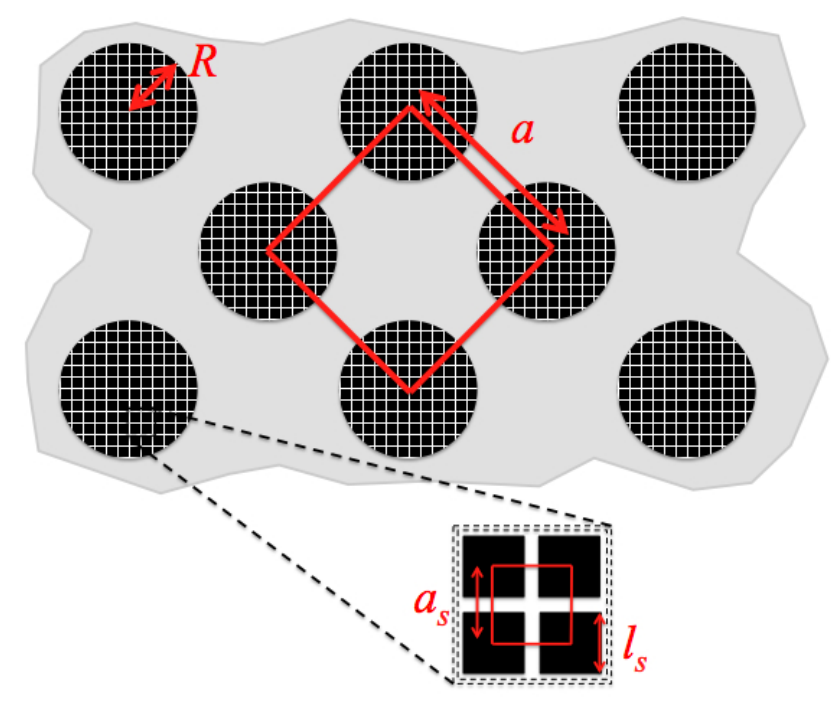

Figure 1. (Color Online) Scheme of the penetrable fluid-fluid system presented in this work. In this example we consider a two dimensional square array with lattice constant $a$ and cylindrical inclusions of radius $R$. The inclusions are clusters of rigid square-rod scatterers with lattice constant $a_{s}$ and length side $l_{s}$.

hand, the scattering problem by an arrangement of penetrable cylinders is analyzed in this work using both the multiple scattering theory (MST) $[2,31]$ and the FEM. In both the eigenvalue and the scattering problems, the continuity of both the pressure and velocity at the boundary of each scatterer is considered. Specifically for the case of the scattering problem, the $T$ matrix for the multiple scattering of a penetrable fluid cylinder can be obtained considering that a refracted wave resides inside the scatterer [31].

\subsection{Dispersion relation and scattering}

Once the basic structure is introduced, in the following we show, numerically and experimentally, that the periodic arrangement of clusters behaves like a periodic fluidfluid system. Notice that the concept of penetrable sonic crystal shown in this work is only restricted by the condition $a>>a_{s}$. So independently of the mesoscopic lattice and the shape of the cluster or the inner periodicity, the concept is valid if the condition $a>>a_{s}$ is accomplished.

The structure presented in the previous section is too complex for an experimental design, since contains a large number of elements. In order to make a simpler experimental arrangement, using a minimum number of elements, we consider a squared shape for the clusters (mesoscopic scatterers), while the small-scale elements (inner scatterers in the cluster) present cylindrical shape as shown in Fig. 2(a).

As a mesoscopic scatterer we consider now a square cluster made of rigid, thin cylindrical rods with radius $r_{s}$, embedded in a fluid as shown in Fig. 2(a). Each cluster is an array of $4 \times 4$ rods, arranged in a square lattice with period $a_{s}=a / 5$, 


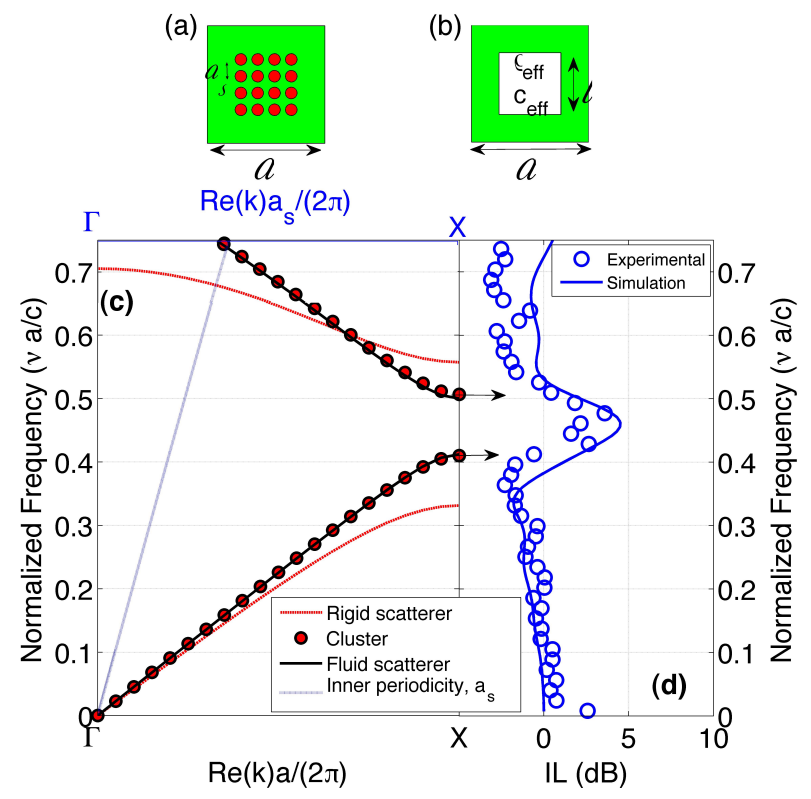

Figure 2. (Color online) (a) Scheme of the unit cell of 2D square array of clusters. (b) Equivalent unit cell from the effective properties of the cluster shown in (a). (c) Band structures in the $\Gamma \mathrm{X}$ direction. Continuous black line (Red dots) represents the band structure for the equivalent fluid-fluid structure (for the periodic arrays of clusters). Dashed line represent the band structures for the case in which the clusters are considered acoustically rigid. Blue dotted lines represent the band structure for the periodicity inside the cluster. We note that for this case the Bloch vector is normalized with respect to $a_{s}$, so the $x$-axis for this band is shown in the upper axis (in blue). (d) Continuous line represent the theoretical IL evaluated at $x=3 a$ of the fluid fluid composite, while dots represent the experimental results.

being the filling fraction $f_{s}=\pi r_{s}^{2} / a_{s}^{2}=0.4$. We notice that at this condition, the wavelength of the Bragg frequency for the mesoscopic scale, governed by $a$, is 5 times bigger than that of the smal inner scale, governed by $a_{s}$. Therefore, considering the long wavelength regime [19], the cluster used here is in the long wavelength regime at normalized frequencies lower than 5/4. In this case we study the behavior of the system up to the normalized frequency equal to 0.75. In this case, the effective parameters of the cluster can be obtained from Eq. (1) and (2). For the particular case in this Section, the effective square scatterer (see Fig. 2(a)) is characterized by $c_{e f f}=0.8452 c_{0}$ and $\rho_{\text {eff }}=2.33 \rho_{0}$. Using these parameters, we calculated the band structure of a square periodic distribution of these effective fluid square scatterers of length side $l=3 a_{s}+2 r_{s}$ with lattice constant $a$ (see Fig. 2(b)), using the PWE method. Black continuous line in Fig. 2(c), represents the dispersion relation, i.e. the band structures calculated from the unit cell shown in Fig. 2(b). They are represented in normalized units with respect to both the lattice constant, $a$, and the properties of the host material. On the other hand, using FEM, we have calculated the band structure of the periodic distribution of the cluster of rigid scatterers, i.e., using the unit cell shown in Fig. 2(a). Red circles in Fig. 2(c) represent this band structure. By comparison of these two results 
we conclude that the periodic distribution of clusters can be considered as a periodic fluid-fluid system. Moreover, in order to highlight the differences between fluid-like, and rigid-fluid composites, we also represent the band structures obtained for rigid square rod scatterers (dashed line) showing the different width of the band gap due to different contrast of impedance. Finally, for the sake of completeness we represent the band structure of the small scale periodic structure inside the cluster (dotted line), showing its linear part at low frequency regime and as a consequence the proof of the validity its homogenization. To have a best comparison with respect to the band structure of the cluster, the Bloch vector was normalized to $a_{s}$, so the $x$-axis for this band is shown in the upper axis (in blue), while the frequencies are normalized to the periodicity of the clusters, $a$.

The structure was designed and its acoustical transmission spectrum (Insertion Loss, IL) was also determined experimentally. Details of the experimental set-up can be found e.g. in Ref. [6]. The IL spectrum of an arrangement of scatterers is obtained measuring transmitted sound pressure levels, with and without the sample, at the same point. $I L$ is determined as

$$
I L=20 \log _{10}\left|\frac{P_{0}(x)}{P(x)}\right|,
$$

where $P_{0}(x)(P(x))$ is the incident pressure (total pressure with the sample) calculated at $x$. In this work $x=3 a$ to avoid the edge diffraction effect.

Figure 2(d) shows the numerical (continuous blue line) and experimental (blue dashed line with open circles) $I L$ of a periodic array of $4 \times 4$ clusters with a unit cell as shown in Fig. 2(a). Note the agreement, in spite of the small number of elements used to build the structure. It shows an increase of the IL at frequencies where a band gap is predicted for the periodic fluid-fluid system. Notice that this attenuation band is narrower than that predicted for the rigid-fluid system (dashed lines in Fig. 2(c)). This supports the assumption that the periodic distribution of clusters of scatterers actually behaves as a periodic fluid-fluid composite.

In what follows we report two acoustical phenomena which are expected to occur only in penetrable structures. Both have been discussed in the photonic case, however a realization in acoustic case may require or may be simplified by using fluid-fluid like structures as those proposed in this work.

\section{Focusing of evanescent waves}

It is commonly accepted that evanescent waves in band gaps lay at the edges of the Brillouin Zone (BZ), i.e. the period of the decaying field oscillations lock to the period of the crystal $[5,7]$. Locking means that, as the frequency changes within the band gap, the real part of the wavevector remains fixed and takes the value at the edge of the BZ (only the phase of the mode is allowed to change). We recently showed [20] that, in photonics, besides the conventional locked evanescent waves, a new class of unlocked evanescent solutions exist in two-dimensional (2D) systems with low index 
contrast. The wave-vectors of these waves have a non zero imaginary part (responsible for the evanescent behaviour), but its real part (denoting its modulation) is not locked to the crystal but it also changes with the frequency within the gap. Additionally, the real part of wavevector shows a curved isofrequency contour, which has been related to different spatial effects on beam propagation [32, 33, 34]. Particularly, for the case of the unlocked evanescent waves one expects that beams propagating at frequencies within the bandgap show focusing, in addition to evanescence. Therefore, these waves could be observed in a low contrast sonic crystal, and find its relevance in thin slabs of periodic materials. The goal of this Section is to show the existence of unlocked evanescent waves in acoustics for penetrable periodic structures, and to show the focusing effect behind thin slabs produced by the unlocked real part of the wave vector. We will consider the analysis of a fluid-fluid structure, having in mind the structure proposed in the previous Section.

\subsection{Complex band structures}

In a recent previous work, the problem of focalization of evanescent fields in a photonic crystal was discussed [20], and the parameters of the structure required to observe the phenomenon were estimated. Here we adapt the previous study to the acoustical case, and consider a square array of fluid cylinders of radius $R$ with properties $\rho_{\text {eff }} / \rho_{0}=12.3$ and $c_{e f f} / c_{0}=0.73$. Figure $3(\mathrm{a})$ shows the solution of the $\omega(\mathbf{k})$ problem using PWE method for the system with a filling fraction $f=\pi(R / a)^{2}=0.63$ in the $\Gamma \mathrm{M}$ direction (see inset of Fig. 3(a)). This solution is shown with continuous black line, and it reveals a band gap in the range $[0.45,0.61]$ in normalized frequency units. Moreover, the complex band structures obtained from the problem $k(\omega)$, shown with blue and red dots, is a bit different. The complex band structures reproduce $(k(\omega)$ problem) the real-valued bands obtained from the $\omega(\mathbf{k})$ but it yields the appearance of additional unlocked evanescent modes in the range of frequencies [0.55, 0.61] (shadowed area in Fig. 3(a)), where the real part of $k$ is not constant, but depends on frequency. A careful inspection of Fig. 3(a) shows that at the lower part of the band gap the evanescent mode remains locked, while increasing the frequency the attenuation rates (imaginary part) of two locked evanescent modes approach and merge, resulting in the appearance of an unlocked evanescent mode (shadowed area in Fig. 3(a)). This kind of evanescent modes connects the closest points of the dispersion curves from two adjacent propagation bands. However, if the frequency maxima and minima from both bands do not lay at high symmetry points of the Brillouin zone, as is the case for the locked evanescent waves, the modes become unlocked and cross the Brillouin zone as shown in Fig. 3(a).

Up to now, all the results have been discussed for the case of real fluid-fluid systems.

Next, we use the periodic system of clusters to analyze its properties. For that, we consider a composite made of a cluster of cylinders with the following filling fractions: $f=0.63$ and $f_{s}=0.8$. The filling fraction $f$ is the same as in the previous fluid-fluid system, shown in Fig. 3(a), and $f_{s}$ reproduces the same sound velocity and density 

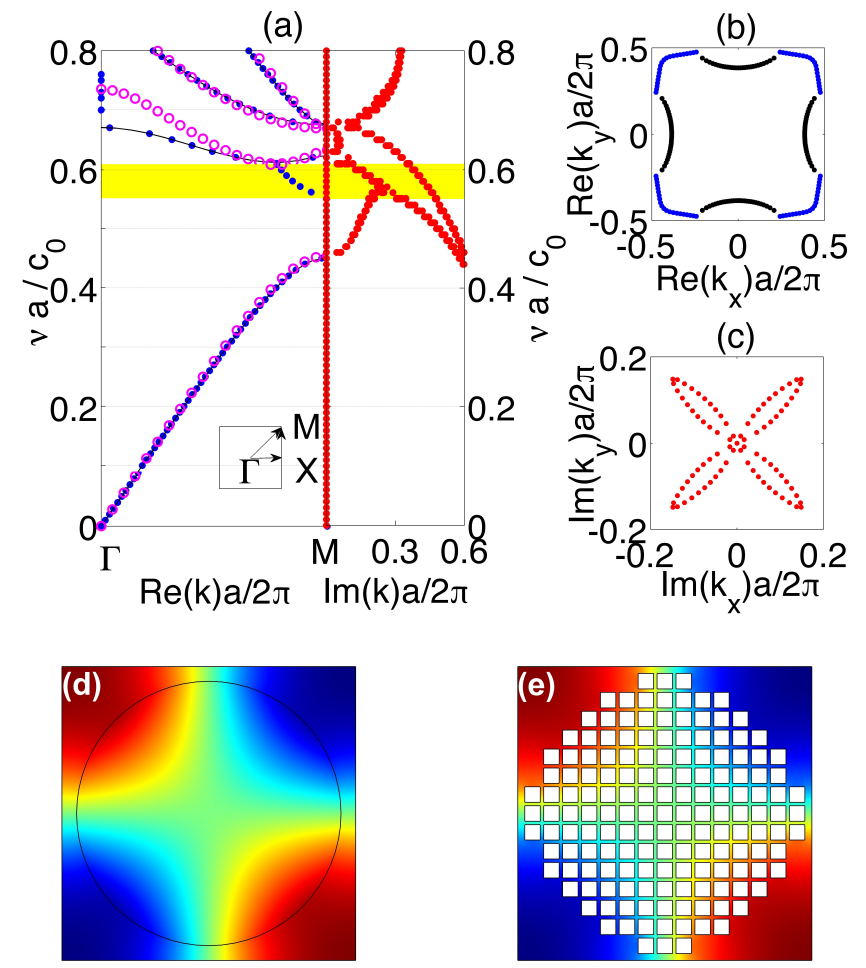

Figure 3. (Color Online) Dispersion relation of a square periodic fluid-fluid system $\left(\rho_{e f f} / \rho_{0}=12.3\right.$ and $\left.c_{e f f} / c_{0}=0.73\right)$. The filling fraction is $f=\pi R^{2} / a^{2}=0.63$. The normalized frequency $\nu a / c_{0}$ and wave-vector $k a / 2 \pi$ are depicted. (a) Complex band structures. Blue dots in left panel show the real part and red dots in the right panel show the imaginary part obtained using the $k(\omega)$-methods (EPWE). For comparison, continuous line in left panel show the band structures obtained using the $\omega(\mathbf{k})$-method (PWE). Purple open circles show the band structures of the systems calculated with the real cluster of rigid cylinders. (b) and (c) show real and imaginary parts of the complex isofrequency contours respectively for the normalized frequency $\nu a / c_{0}=0.58$. Black dots represents pure real eigenvectors, while blue and red dots represent the real and the imaginary parts of the complex wave-vectors respectively. (d) and (e) show the pressure field in the unit cell for the eigenvalue of the first band at point $M$ $\left[\left(k_{x}, k_{y}\right)=(\pi, \pi)\right]$ calculated with the equivalent fluid-fluid system and with the real cluster of rigid cylinders respectively.

ratio as for the fluid-fluid system. Using the square rod scatterers, arranged in a square periodicity inside the cluster, we construct a quasi cylindrical inclusion (see Fig. 3(e) for the unit cell) with the desired physical properties. At this stage, we check that the periodic distribution of clusters reproduces the same properties as the fluid-fluid composite previously analyzed. Purple open dots in Fig. 3(a) show the band structures calculated from the $\omega(\mathbf{k})$ problem for the system made of clusters. Notice that the band structure calculated with the fluid-fluid composite and that corresponding with the periodic distribution of clusters are in good agreement. The small discrepancies in the second band around the $\Gamma$ point are due to the non perfect cylindrical shape of the cluster. Figures 3(d) and 3(e) show the pressure field in the unit cell for the eigenvalue of the first band at point $\mathrm{M}\left[\left(k_{x}, k_{y}\right)=(\pi, \pi)\right]$ calculated with the fluid scatterer and 

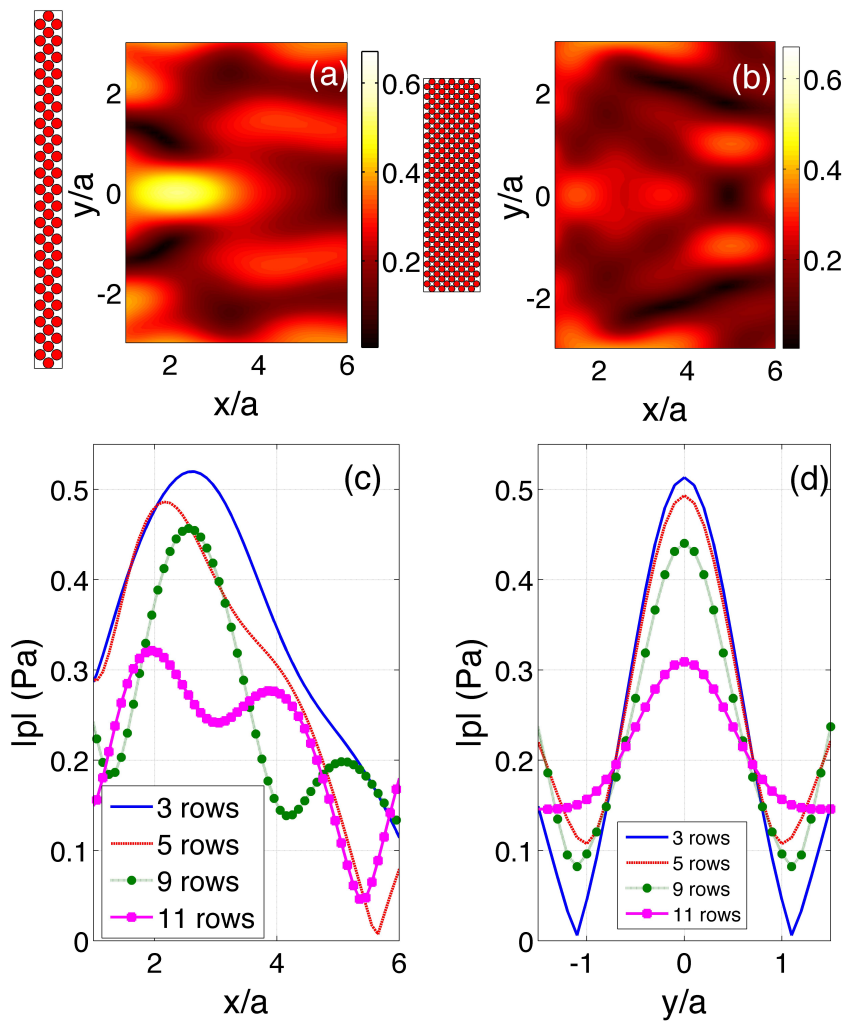

Figure 4. (Color Online) Transmission properties of several periodic slabs of the fluid-fluid system predicted by MST. (a) and (b) show the pressure field, $|p|$, for two slabs of 3 rows and 11 rows. (c) and (d) show the longitudinal and transversal cuts along the $x$-axis and $y$-axis respectively of (a) for different slabs with different number of columns.

with the cluster of rigid square-rod scatterers respectively. The agreement between the two eigen-fields is good.

In order to study the spatial dispersion relation and the effect of the non zero real part of the unlocked evanescent waves in the band gap, we have analyzed the complex isofrequency contours for the central frequency of the unlocked evanescent mode. Figures 3(b) and 3(c) show the real and the imaginary part of the complex isofrequency contours at the normalized frequency $\nu a / c_{0}=0.58$ respectively. Note that the real part of the isofrequency contour presents, along the $\Gamma \mathrm{M}$ (diagonal) direction, a region in which the curvature is positive. Positive curvatures in $k$-space (the convex case) introduce negative diffraction inside the periodic medium, which is afterwards compensated at some distance after the crystal by normal (positive) diffractive propagation in the surrounding fluid $[32,33,34]$. That distance is the focalization distance, so the system acts as a flat lens. Then, waves constructed from such unlocked evanescent modes can have a sufficient propagation freedom in order to develop curved wave-fronts. Then, we expenct that evanescent waves can be focused behind a thin flat-flat interface slice of periodic material. 


\subsection{Focusing of unlocked evanescent waves}

The predicted focusing of evanescent beams is now demonstrated by direct numerical simulations using MST. The positive curvature of the real part of the isofrequency contours along the $\Gamma \mathrm{M}$ direction indicates that focusing of an evanescent beam behind a thin fluid-fluid system can be expected. To observe that, we consider a line source placed $5 a$ from the crystal and we evaluate the acoustic field behind several slabs with different number of rows.

Figures 4(a) and 4(b) show the acoustic pressure field, $|p|$, behind two slabs with 3 and 11 rows respectively. One can clearly see both the focusing behaviour for the case of 3 rows and the decay of the amplitude of the focus as we increase the number of rows in the crystal, obviously because the effect is mediated by evanescent waves. During the process of calculation we have analyzed the acoustic profile of structures made of different number of columns and we have evidenced that the effect of focusing by these structures is not an edge diffraction effect but arising from the accumulation of phase-shift by the evanescent wave inside the crystal which is compensated outside the crystal creating the focus. Figures $4(\mathrm{c})$ and $4(\mathrm{~d})$ show the longitudinal and transversal cuts for different slabs with several number of rows. One clearly sees that an increasing of the number of rows implies a decreasing of the intensity of the focus.

\section{Coupled resonator acoustical waveguide (CRAW)}

The guiding of waves in periodic structures, making use of the presence of the band gap, is relevant in different applications in electromagnetism, optics and acoustics. As an example, a photonic crystal waveguide, formed by a line defect inside the crystal, can guide the light with high transmission efficiency in the sub-micron scale [7], which is very difficult to achieve for a dielectric waveguide. However due to the strong dependence of the band gap on the geometry of the system, the waveguiding using these mechanisms presents some disadvantages. A strong bend of the waveguide in the periodic structure implies large interior reflections and, as a consequence, a reduction of the efficiency of the waveguide. Moreover, the path of the waveguide must follow the specific lattice orientation of the periodic background and should be embedded in a wide enough periodic media to avoid the propagation loss, so the waveguide occupies much space in the transverse dimension $[21,22]$.

A different type of optical waveguide named coupled resonator optical waveguide (CROW) has been proposed [23]. A CROW consists of an array of coupled resonators with high quality factor (Q-factor), and the guiding of light is due to photon hopping [35] along the successive resonators. In this Section, we study the transmission properties of an alternative kind of waveguides based on the distribution of penetrable acoustic scatterers that use index guiding mechanism [23]. The waveguide is formed by aligning a 1D array of penetrable scatterers in a fluid medium. This can be considered as an acoustic analogue of the CROW, so we call the system a coupled resonator acoustical 

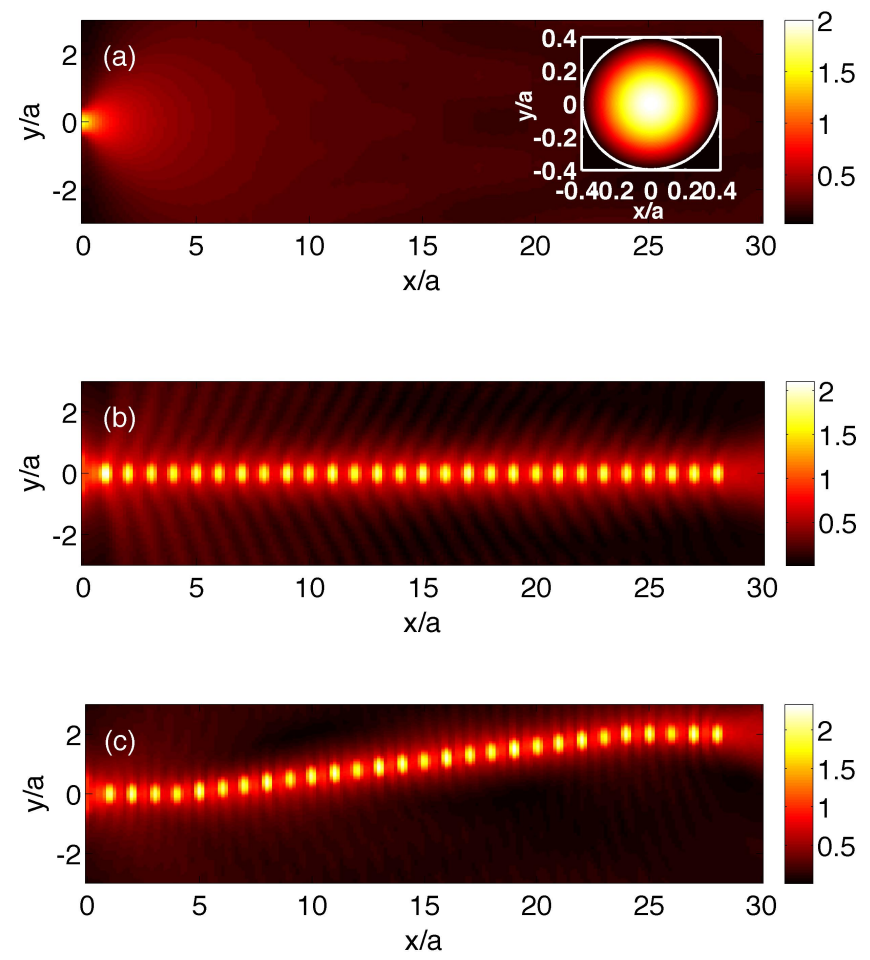

Figure 5. (Color online) (a) Incident acoustic field at $\nu a / c_{\text {host }}=0.78$. Inset shows the resonant mode of the fluid scatterer at $\nu a / c_{\text {host }}=0.78$. (b) and (c) Guided waves at the resonant frequency, $\nu a / c_{\text {host }}=0.78$, by a straight and a curved discrete distribution of 28 penetrable scatterers respectively. Color scale show the values of $|p|$.

waveguide (CRAW).

First, we analyze a 1D arrangement of fluid-like cylindrical clusters which properties are fixed by the inner filling fraction, $f_{s}=0.5$, therefore $c_{\text {eff }} / c_{\text {host }}=0.82$ and $\rho_{\text {eff }} / \rho_{\text {host }}=3.04$. Here we consider a periodic row of penetrable scatterers. We excite the waveguide with the acoustic field shown in Fig. 5(a) at the normalized frequency $\nu a / c=0.78$, which corresponds to the first resonance of the penetrable scatterer (shown in the inset of Fig. 5(a)). The case of the periodic waveguide is shown in Fig. 5(b).

We next show the possibility of guiding a wave through bended paths using a CRAW. Without loss of generality, we analyze a waveguide that has a input at $y / a=0$ and the output is at $y / a=2$ as shown in Fig. 5(c). Figure 5(c) shows the transmission through the waveguide by the activation of the index guiding mechanism for the first resonant frequency.

We can see in both cases that at the excitation frequency, the resonance of the penetrable scatterers is excited through the waveguide so the index guiding mechanism is activated and waves are transmitted through the waveguide. It is worth noting here that, as in the case of the optical counterpart (CROW), we assume sufficiently large separation between the individual resonators that the resonators has a weakly near field coupling. Consequently, we expect that the transmitted mode in such a coupled- 
resonator waveguide remains essentially the same as the mode in a single resonator. At the same time, the far field coupling must be taken into account. Such coupling is based on evanescent-field coupling, between the individual modes to explain the transmission of waves. This coupling is an analogue of the tight-binding limit in condensed-matter physics, [36] in which the overlap of atomic wave functions is large enough that corrections to the picture of isolated atoms are required. The individual resonators in our waveguide are the analogue of the isolated atoms, and the resonant mode in the resonators corresponds to the atomic wave function.

\section{Concluding remarks}

We have proposed a periodic structure made of penetrable scatterers for scalar waves, the penetrable sonic crystals. The approach is based on the fluid-like behaviour of a cluster made of rigid scatterers. These clusters have their physical properties managed by the filling fraction of the micro-structure. Therefore, we propose here a double periodicity composite made of a periodic distribution of fluid-like clusters; one periodicity corresponds to large scale arrangement of the clusters, and the other periodicity corresponds to the small scale inside the cluster. Therefore the inner periodicity controls the physical properties of the fluid-like scatterer and the size and the distribution of the clusters the propagation properties of the penetrable sonic crystals. Analytical and numerical simulations show that the periodic distribution of clusters embedded in fluid is equivalent to the case of fluid-fluid periodic system with penetrable scatterers. Using this main property, we show here that the proposed structure can be used to observe the presence of unlocked evanescent waves and to design a coupled resonator waveguide for scalar waves. These fluid-fluid systems could in practice pave the way to show several wave control phenomena in different branches of science and technology and extensions of this work can be developed to analyze periodic structures made of anisotropic scatterers.

\section{Acknowledgments}

We acknowledge financial support by Spanish Ministerio de Economia y Competitividad and European Union FEDER through project FIS2011-29731-C02-01 and -02.

\section{References}

[1] W.C. Chew. Waves and Fields in inhomogeneous media. (IEE Press, New York, 1995).

[2] Martin P., Multiple Scattering. Interaction of Time-Harmonic Waves with N Obstacles (Cambridge University Press, UK, 2006).

[3] Maurel A., Mercier, J.-F. and Felix, S. J. Acoust. Soc. Am. 135, 165, (2014).

[4] R. Martínez-Sala, J. Sancho, J. V. Sánchez, V. Gómez, J. Llinares, and F. Meseguer. Nature, 378, 241, (1995).

[5] Brillouin L., Wave Propagation in Periodic Structures, 2nd Edition. (Dover, New York, 1953). 
[6] Romero-García, V.; Sánchez-Pérez, J. V.; Garcia-Raffi, L. M.; Herrero, J. M.; García-Nieto, S. and Blasco, X. J. Acoust. Soc. Am., 125 (6), 3774-3783, (2009).

[7] Joannopoulos J., Johnson S., Winn J. and Meade R., Photonic Crystals. Molding the Flow of Light, (Princeton, University press, 2008).

[8] Sigalas, M.; Kushwaha, M. S.; Economou, E. N.; Kafesaki, M.; Psarobas, I. E. and Steurer, W. Z. Kristallogr., 220, 765-809, (2005).

[9] Pennec, Y.; Vasseur, J. O.; Djafari-Rouhani, B.; Dobrzyski, L. and Deymier, P. A. Surface Science Reports, 65, 229-291, (2010).

[10] Sánchez-Pérez, J. V.; Caballero, D.; Martínez-Sala, R.; Rubio, C.; Sánchez-Dehesa, J.; Meseguer, F.; Llinares, J. and Gálvez, F. Phys. Rev. Lett., 80, 5325-5328, (1998).

[11] Maurel A., Mercier, J.-F. and Felix, S. Phys. Rev. B 88, 115416, (2013).

[12] Cai, L.W., Dacol, D.K., Calvo, D.C., Orris, G.J. J. Acoust. Soc. Am. 122, 1340, (2007).

[13] Maurel A., Mercier, J.-F. J. Acoust. Soc. Am. 131, 1874, (2012).

[14] Feng-Lian Li, Yue-Sheng Wang and Chuanzeng Zhang. Phys. Scr. 84, 055402, (2011).

[15] Allard, J.-F. and Atalla, N. Propagation of Sound in Porous Media: Modelling Sound Absorbing Materials, Second Edition. (Willey, United Kingdom, 2009).

[16] Umnova, O.; Attenborough, K. and Linton, C. M. J. Acoust. Soc. Am., 119, 278, (2006).

[17] Barryman J. G.,J. Acoust. Soc. Am.,68,1809, (1980).

[18] Mei J., Liu Z., Wen W. and Sheng P.,Phys. Rev. Lett. 96, 024301, (2006).

[19] Torrent D. and Sanchez-Dehesa J., Phys. Rev. B 74, 224305, (2006).

[20] Botey M., Cheng Y.-C., Romero-García V., Picó R., Herrero R., Sánchez-Morcillo V. and Staliunas K.,Opt. Lett. 38, 11, 1890-1892 (2013) .

[21] M. Zhang, W. Zhong and X. Zhang. J. Appl. Phys., 111, 104314, (2012).

[22] J.M. Escalante, A. Martínez and V. Laude. J. Phys. D. Appl. Phys., 46, 475301, (2014).

[23] Yariv, A., Xu, Y., Lee, R.K., Scherer, A. Coupled resonator optical waveguide: a proposal and analysis. Opt. Lett. 24, 711, (1999).

[24] Rockstuhl C., Lederer F., Etrich C., Pertsch T. and Scharf T., Phys. Rev. Lett. 99, 017401, (2007).

[25] Yannopapas V. and Vanakaras A. G.,Phys. Rev. B 84, 085119, (2011).

[26] Kushwaha, M.; Halevi, P.; Martnez, G.; Dobrzynski, L. and Djafari-Rouhani, B. Phys. Rev. B, 49, 2313, (1994).

[27] Romero-García, V.; Sánchez-Pérez, J. and Garcia-Raffi, L. J. Appl. Phys., 108, 044907, (2010).

[28] Laude, V., Achaoui, Y., Benchabane, S. and Khelif, A. Phys. Rev. B, 80, 092301, (2009).

[29] Ihlenburg F., Finite Element Analysis of Acoustic Scattering (Springer Verlag, New York Inc., 1998).

[30] Rongzhou Wang, Xue-Hua Wang, Ben-Yuan Gu, and Guo-Zhen Yang. J. Appl. Phys. 90, 4307 (2001).

[31] Chen Y. and Ye Z., Phys. Rev. E 64, 036616, (2001). Chen Y. and Ye Z.,Phys. Rev. Lett. 87, 1843011, (2001).

[32] Notomi M.,Phys. Rev. B 62, 10696, (2000).

[33] Kosaka H., Kawashima T., Tomita A., Notomi M., Tamamura T., Sato T. and Kawakami S., Appl. Phys. Lett. 74, 1212, (1999).

[34] Pérez-Arjona I., Sánchez-Morcillo V. J., Redondo J., Espinosa V. and Staliunas K.,Phys. Rev. B 75 (2007) 014304.

[35] M. Bayindir, B. Temelkuran, and E. Ozbay, Phys. Rev. B 61, R11855-8 (2000).

[36] N. W. Ashcroft and N. D. Mermin, Solid State Physics (Saunders, Philadelphia, Pa., 1976). 